\title{
BETA CAROTENE AND HESPERIDIN ANTIOXIDANTS MITIGATE HEPATOTOXIC EFFECTS OF IMIDACLOPRID IN MALE RATS.
}

\author{
Bashandy, S.A. ${ }^{\text {; }}$ Bashandy, M.A. ${ }^{2}$; El Zawahry, E.I. ${ }^{2}$; Adly, F. ${ }^{\text {; }}$ Abdel Naby, M.F. ${ }^{2}$ \\ ${ }^{1}$ Department of Pharmacology, Medical Division, National Research Centre, \\ 33 EL Bohouth st., Dokki, Cairo Governorate, Egypt. P.O.12622 \\ ${ }^{2}$ Zoology Department, Faculty of Science, Al-Azhar University, Egypt. \\ ${ }^{3}$ Pathology Department, National Research Center, Egypt.
}

\begin{abstract}
The role of beta carotene $(20 \mathrm{mg} / \mathrm{kg})$ and hesperidin $(100 \mathrm{mg} / \mathrm{kg})$ in protection against imidacloprid $(45$ $\mathrm{mg} / \mathrm{kg}, 90 \mathrm{mg} / \mathrm{kg}$ that represent $1 / 10 \mathrm{LD} 50$ and $1 / 5$ LD 50 of IM) induced hepatotoxicity was investigated. All the treatments were given orally and daily for thirty days.

The treatment of rats with IM showed a significant increase in plasma aspartate aminotransferase (AST), alanine aminotransferase (ALT), alkaline phosphatase (ALP), Gamma-glutamyltransferase (GGT), alpha fetoprotein $(\alpha-F P)$, total bilirubin, total cholesterol $(T C)$ and triglycerides (TG). Also, Imidacloprid treatment led to a significant rise of hepatic malondialdehyde (MDA), tumor necrosis factor-alpha (TNF- $\alpha)$ and nitric oxide (N.O). On the other-hand, plasma total protein (TP) and albumin tended to a significant decrease due to IM treatment. Hepatic catalase, reduced glutathione (GSH) and superoxide dismutase (SOD) of IM group exhibited a significant decrease. The histological study showed lesions in the liver of treated rats with IM. Both beta carotene and hesperidin mitigate the deleterious effects of IM on previous parameters as manifested by a significant decrease of MDA, N.O, liver enzymes, and improved antioxidant enzyme levels and liver histology. The protective effect of beta carotene and hesperidin in combination is more pronounced. Therefore, our results clarify the synergistic effect of these two antioxidants on alleviating imidacloprid toxicity.
\end{abstract}

Key words: Imidacloprid, beta- carotene, hesperidin, liver function, oxidative stress, synergistic effect, hepatoprotection.

\section{INTRODUCTION}

Imidacloprid (IM) is a member of a group of insecticides called neonicotinoids. The extensive use of insecticides has been reviewed in recent years due to their stability in the environment and their accumulation in the living tissues of organisms. Imidacloprid is extensively used in agriculture for control of the sucking insects and also used as a foliar treatment for soil and for seed dressing (Felsot, 2001). In Veterinary Medicine, it is used as flea control agent on dogs and cats (Hutchinson et al., 2001). IM is most widely used neonicotinoids insecticide in agriculture (Whitehorn et al., 2012). Such large scale as can magnify the toxic properties and adverse effects of insecticide that affect on human as well as animal health. The toxicity of IM following ingestion showed clinical effects such as nausea, vomiting, respiratory failure, seizures and even death in human (Agarwal \& Srinivas, 2007 and Proenca et al., 2005). IM can impair the liver and kidney functions (Arfat et al., 2014). IM evokes hepatic oxidative stress as confirmed by the increase of lipid peroxidation and a decrease of reduced glutathione (Ahmed and Nasr, 2015). It was found that the toxicity of imidacloprid can originate from free radicals production by the cell which causes an oxidative stress and tissue damages (El-Gendy et al., 2010). These damages modified macromolecules such as nucleic acids, lipids, and proteins, which result in functional changes of the target cell (Stephan et al., 1997).

Antioxidants can eliminate free radicals and other reactive oxygen and nitrogen species, and these reactive species contribute to most chronic diseases. The natural antioxidants in foods may induce antioxidant defense mechanisms (Kensler et al., 2007). Phytochemicals, which are found in fruits, vegetables, and plant-derived beverages, may act as cytoprotective agents in many organs 
(Orhan et al., 2011). Naturally occurring carotenoids and flavonoids possess free radical scavenging properties and offer protection from oxidative injury (Ansari et al., 2008). Beta Carotene (BC), a pigment naturally found in many fruits and vegetables, has one of the highest antioxidant potentials found among carotenoids (Stahl and Sies, 2003). BC enhances antioxidant defense system (Novo et al., 2013). BC ameliorates the deleterious effects of acetaminophen (Morakinyo et al., 2012) and aflatoxin (Kheir-Eldin et al., 2008) on liver tissues.

Hesperidin, a flavanone glycoside is present richly in citrus fruits or in its peel (Levaj et al., 2009). Hesperidin (HSP) has been reported to have many biologically important properties including antioxidant (Wilmsen et al., 2005), hypoglycemic, hypolipidemic (Akiyama et al., 2009), antiinflammatory, analgesic (Vabeiryureilai et al., 2015), and anticancer activities (Devi et al., 2015). HSP protects against methionine induced oxidative stress and neurotoxicity (Kumar et al., 2017), diethylnitrosamin induced nephrotoxicity (Ahmed et al., 2015), and doxorubicin induced hepatotoxicity (Hozayen et al., 2014). It was suggested that hesperidin exhibited its protective effect against cyclophosphamide -induced hepatotoxicity through upregulation of hepatic peroxisome proliferator activated receptor gamma expression and the abolition of inflammation and oxidative stress (Mahmoud, 2014). The mixture of antioxidants can act synergistically result in more efficient protection (Shi et al., 2004). The present study aims to examine the antioxidant activity of beta carotene and hesperidin (separately or in combination) against imidacloprid- induced hepatotoxicity and oxidative stress.

\section{MATERIAL AND METHODS}

\section{Chemicals:}

Imidacloprid of $96 \%$ purity produced by the Bayer (Germany) was used in the present experiments. Beta Carotene (BC) was obtained from El-Goumhouria Co. for Trading
Medicine, Cairo, Egypt. Hesperidin was obtained from Sigma, USA.

\section{Animals:}

Fifty- four adult male albino rats (Rattus norvegicus), weighing 140 - 160 g. were used in the present study. The rats were purchased from the animal house, National Research Centre, Egypt. (Dokki, Giza, Egypt). They were kept for one week for acclimatization before the experiment. They were housed in standard polypropylene cages and kept under thermostatically controlled room of 12-h light/dark cycle. They were maintained on standard pellet diet with free access to water.

\section{Ethics statement}

This experiment was carried out in according to the recommendations in the Guide for the Care and Use of Laboratory Animals of the National Institutes of Health (NIH publication No. 85-23, revised 1996) and under regulations of Animal Care and Use of National Research Centre in Egypt. All sampling was performed under light anesthesia with ether.

\section{Experimental design:}

Rats were randomly divided into the following groups:

Group I Considered as normal control $(\mathrm{n}=6$ rats).

\section{Group II}

A- Animal received a daily low dose of imidacloprid (45 mg/kg b.w., 1/10 $\mathrm{LD}_{50}$ ) orally for 30 days ( $\mathrm{n}=6$ rats).

B- Animal received a daily high dose of imidacloprid (90 mg/kg b.w., $1 / 5 \mathrm{LD}_{50}$ ) orally for 30 days ( $n=6$ rats).

\section{Group III}

A- The rats were treated daily with a low dose of imidacloprid ( $45 \mathrm{mg} / \mathrm{kg}$ b.w.) and beta carotene at a dose level of $20 \mathrm{mg} / \mathrm{kg}$ b.w. (Cui et al., 2012) orally for 30 days ( $n=6$ rats).

B- The animals received a daily high dose of imidacloprid $(90 \mathrm{mg} / \mathrm{kg} \mathrm{b.w.)}$ and beta carotene $(20 \mathrm{mg} / \mathrm{kg}$ b.w.) orally for 30 days $(\mathrm{n}=$ 6 rats).

\section{Group IV}


A- The rats received a daily low dose of imidacloprid ( $45 \mathrm{mg} / \mathrm{kg} \mathrm{b.w.)}$ and hesperidin at the dose of $100 \mathrm{mg} / \mathrm{kg}$ b.w. (Kumar et al., 2017) orally for 30 days ( $n=6$ rats).

B- Each animal received a daily high dose of imidacloprid $(90 \mathrm{mg} / \mathrm{kg}$ b.w.) and hesperidin $(100 \mathrm{mg} / \mathrm{kg}$ b.w.) orally for 30 days ( $\mathrm{n}=6 \mathrm{rats})$.

\section{Group V}

A- Each animal received a low dose of imidacloprid orally after administration of beta carotene and hesperidin in combination daily for 30 days ( $n=6$ rats).

B- Each animal received a daily high dose of imidacloprid orally after administration of beta carotene and hesperidin in combination for 30 days ( $\mathrm{n}=6$ rats).

Both IM and HSP were dissolved in water, while $\beta C$ was dissolved in corn oil.

\section{Determination of imidacloprid LD50 in rats}

The oral $\mathrm{LD}_{50}$ determination was done by the method of Lorke (1983).

\section{Samples collection:}

At the end of the experiment, blood was withdrawn from retro- orbital plexus of the eye and collected in glass centrifuge tubes and allows it to clot at room temperature for thirty minutes. Blood samples were centrifuged at $4000 \mathrm{rpm}$, for $10 \mathrm{~min}$, and serum was separated into eppendorph tubes and stored at $-20 \mathrm{C}$ till used for biochemical analysis. The liver were removed and fixed in $10 \%$ formol saline for histological examination.

\section{Biochemical analysis}

Total protein, albumin, AST, ALT, ALP, GGT, $\alpha$-FP ,total bilirubin, total cholesterol, and triglycerides were determined in serum by chemistry analyzer, Beckman Coulter- Model AU 480, Full automatic, Japan, using Roche kits. Moreover, MDA, SOD, catalase, GSH, and N.O were determined colorimetrically in liver tissues (Photometer-Model 5010) using commercial kits produced by Biodiagnostic, Egypt. ELISA technique was carried out for the assay of tumor necrosis factor-alpha ( $\mathrm{R}$ and $\mathrm{D}$ Systems, USA).

\section{Histological evaluation:}

Slices of liver tissue were fixed in $10 \%$ formol saline, and processed for preparation of paraffin blocks. Sections of 5-7 $\mu$ in thickness were cut and stained with Hematoxylin \& Eosin (H\&E) stain.

\section{Statistical analysis:}

The data were analyzed using the one-way analysis of variance (ANOVA) followed by Fisher's least significant difference (LSD) analysis to compare various groups with each other. Results were expressed as a mean \pm standard error (SE).

\section{RESULTS:}

\section{Biochemical parameters:}

It was observed that hepatic catalase, GSH, and SOD levels of rats treated with IM decreased significantly $(\mathrm{P} \leq 0.05)$ in comparison with control (Table 1). On the other hand, hepatic MDA, N.O and TNF- $\alpha$ concentrations of IM group elevated significantly. Although, hepatic SOD, CAT, and GSH of BC+IM or $\mathrm{H}+\mathrm{IM}$ groups lowered significantly as compared with control, their values were higher significantly than those of IM group. Both beta carotene and hesperidin reduced the effects of IM on hepatic oxidative stress parameters.

The results demonstrated a significant increase of AST, ALT, ALP, GGT and total bilirubin (Table2) in all treated groups. The values of liver enzymes and bilirubin levels of beta carotene + IM or hesperidin + IM groups were significantly lower than those of IM group. Similar results were obtained in the case of alpha-fetoprotein.

The total protein and albumin levels in serum of IM group showed a significant decrease $(\mathrm{P} \leq 0.05)$, while total cholesterol and triglycerides levels exhibited a significant increase $(\mathrm{P} \leq 0.05)$ as compared with control (Table 3). Both beta carotene and hesperidin alleviated the effects of IM on previous parameters.

\section{Histological results of the liver:}

The liver of control rats revealed the normal characteristic architecture of hepatic lobules and central vein (Fig. 1A). In the case of liver sections treated with $1 / 5 \mathrm{LD}_{50}$ of 
imidacloprid insecticide showing dilated, congested, edematous, vacuolated portal vein with infiltration of leukocytes and a fibrosis around the bile duct. Dilated and congested blood sinusoid, sings of degeneration in the form of karyolysis, karyorrhexis and foci of necrosis were seen (Figs. 1B, C, D).

The microscopic examination of liver tissue of a rat subjected to $1 / 10 \mathrm{LD}_{50}$ of imidacloprid insecticide showing hepatocytes with homogenous cytoplasm, congested of portal vein and thickening of the wall and vacuolar degeneration. Dilated blood sinusoids, foci of necrosis and karyolysis (Figs. 2A, B).

Concerning rats treated with $1 / 5 \mathrm{LD}_{50}$ with imidacloprid along with beta carotene showing normal histological structure of hepatic lobules but mild dilated of blood sinusoid and vacuolar degeneration could be observed (Fig. 2C). The liver of rats treated with $1 / 10 \mathrm{LD}_{50}$ with imidacloprid along with beta carotene showing a normal histological structure of hepatic lobules but mild dilated and congestion of blood sinusoid and minute vacuolar degeneration could be observed (Fig. 2D).

Microscopic examination of liver tissue from of rats treated with $1 / 5 \mathrm{LD}_{50}$ with imidacloprid along with hesperidin showing vacuolar degeneration, dilated blood sinusoid and dilated and congested blood vessel (Fig.3A). The hepatocyte of a rat treated with $1 / 10 \mathrm{LD}_{50}$ of imidacloprid insecticide along with hesperidin showing most of hepatocyte appeared normal and few appeared necrotic and congested blood vessel (Fig. 3B).

In the case of the liver of a rat treated with $1 / 5 \mathrm{LD}_{50}$ of imidacloprid insecticide along with beta carotene and hesperidin showing most of hepatocyte appeared normal but necrosis of some hepatocyte (thickening in the wall of portal vessel, hypertrophied of kupffer cells (Fig. 3C). Concerning rats treated with $1 / 10$ $\mathrm{LD}_{50}$ with imidacloprid along with hesperidin and beta carotene showing most of the hepatocyte appeared normal but minute vacuolar degeneration still present (Fig. 3D).

Table (1): Protective effect of Beta Carotene and Hesperidin antioxidants against Imidacloprid insecticide induces alteration in hepatic MDA, CAT., GSH, SOD and N.O. concentration.

\begin{tabular}{|c|c|c|c|c|c|c|c|c|c|}
\hline \multirow{3}{*}{ Group } & \multirow{3}{*}{$\mathrm{CO}$} & & & \multicolumn{6}{|c|}{ Antioxidants and Insecticide } \\
\hline & & \multicolumn{2}{|c|}{ IM } & \multicolumn{2}{|c|}{$\mathrm{BC}+\mathrm{IM}$} & \multicolumn{2}{|c|}{$\mathrm{H}+\mathrm{IM}$} & \multicolumn{2}{|c|}{$\mathrm{BC}+\mathrm{H}+\mathrm{IM}$} \\
\hline & & $1 / 10 \mathrm{LD}_{50}$ & $1 / 5 \mathrm{LD}_{50}$ & $1 / 10 \mathrm{LD}_{50}$ & $1 / 5 \mathrm{LD}_{50}$ & 1/10 LD 50 & $1 / 5 \mathrm{LD}_{50}$ & 1/10 LD 50 & $1 / 5 \mathrm{LD}_{50}$ \\
\hline $\begin{array}{c}\text { MDA } \\
(\mathrm{nmol} / \mathrm{g})\end{array}$ & $\begin{array}{r}21.13 \\
\pm 1.51 \\
\end{array}$ & $\begin{array}{c}* \\
31.27 \\
\pm 1.32 \\
\end{array}$ & $\begin{array}{c}* \\
41.85 \\
\pm 1.37 \\
\end{array}$ & $\begin{array}{c}a \\
23.60 \\
\pm 3.00 \\
\end{array}$ & $\begin{array}{c}* a \\
27.27 \\
\pm 2.10 \\
\end{array}$ & $\begin{array}{c}a \\
17.17 \\
\pm 3.38 \\
\end{array}$ & $\begin{array}{c}\mathrm{a} \\
18.87 \\
\pm 2.89 \\
\end{array}$ & $\begin{array}{c}a \\
17.17 \\
\pm 1.74 \\
\end{array}$ & $\begin{array}{c}\mathrm{a} \\
23.47 \\
\pm 1.69 \\
\end{array}$ \\
\hline $\begin{array}{c}\text { CAT } \\
(\mathrm{U} / \mathrm{g} . \text { tissue })\end{array}$ & $\begin{array}{r}1.84 \\
\pm 0.02 \\
\end{array}$ & $\begin{array}{c}* \\
0.68 \\
\pm 0.08 \\
\end{array}$ & $\begin{array}{c}* \\
0.53 \\
\pm 0.08 \\
\end{array}$ & $\begin{array}{c}* a \\
1.40 \\
\pm 0.08 \\
\end{array}$ & $\begin{array}{c}* a \\
1.16 \\
\pm 0.10 \\
\end{array}$ & $\begin{array}{c}* a \\
1.05 \\
\pm 0.09 \\
\end{array}$ & $\begin{array}{c}* a \\
0.87 \\
\pm 0.07 \\
\end{array}$ & $\begin{array}{c}* a b c \\
1.62 \\
\pm 0.04 \\
\end{array}$ & $\begin{array}{c}* a b c \\
1.54 \\
\pm 0.08 \\
\end{array}$ \\
\hline $\begin{array}{c}\text { GSH } \\
\text { (mg/g. tissue) }\end{array}$ & $\begin{array}{r}4.57 \\
\pm 0.41 \\
\end{array}$ & $\begin{array}{c}* \\
1.87 \\
\pm 0.22 \\
\end{array}$ & $\begin{array}{c}* \\
1.50 \\
\pm 0.34 \\
\end{array}$ & $\begin{array}{c}* a \\
3.12 \\
\pm 0.33 \\
\end{array}$ & $\begin{array}{c}* a \\
2.83 \\
\pm 0.31 \\
\end{array}$ & $\begin{array}{c}* \\
2.50 \\
\pm 0.23 \\
\end{array}$ & $\begin{array}{c}* \\
2.22 \\
\pm 0.27 \\
\end{array}$ & $\begin{array}{c}* a c \\
3.43 \\
\pm 0.33 \\
\end{array}$ & $\begin{array}{c}* a c \\
3.27 \\
\pm 0.33 \\
\end{array}$ \\
\hline $\begin{array}{c}\text { SOD } \\
\text { (U/gm tissue) }\end{array}$ & $\begin{array}{r}397.40 \\
\pm 16.95 \\
\end{array}$ & $\begin{array}{c}* \\
281.52 \\
\pm 13.14 \\
\end{array}$ & $\begin{array}{c}* \\
268.40 \\
\pm 17.30 \\
\end{array}$ & $\begin{array}{c}* \\
298.78 \\
\pm 18.83 \\
\end{array}$ & $\begin{array}{c}* \\
290.03 \\
\pm 20.42 \\
\end{array}$ & $\begin{array}{c}\text { *a } 335.15 \\
\pm 21.83\end{array}$ & $\begin{array}{c}* \\
317.77 \\
\pm 23.75 \\
\end{array}$ & $\begin{array}{c}\mathrm{ab} \\
370.02 \\
\pm 18.72 \\
\end{array}$ & $\begin{array}{c}\mathrm{ab} \\
356.02 \\
\pm 14.71 \\
\end{array}$ \\
\hline $\begin{array}{c}\text { N.O. } \\
\text { (nmol/L) }\end{array}$ & $\begin{array}{r}1.60 \\
\pm 0.13\end{array}$ & $\begin{array}{c}* \\
2.20 \\
\pm 0.14\end{array}$ & $\begin{array}{c}* \\
2.50 \\
\pm 0.17\end{array}$ & $\begin{array}{c}a \\
1.78 \\
\pm 0.13\end{array}$ & $\begin{array}{c}\mathrm{a} \\
1.85 \\
\pm 0.17\end{array}$ & $\begin{array}{r}1.80 \\
\pm 0.15\end{array}$ & $\begin{array}{c}a \\
2.00 \\
\pm 0.17\end{array}$ & $\begin{array}{c}\text { abc } \\
1.39 \\
\pm 0.13\end{array}$ & $\begin{array}{c}\mathrm{a} \\
1.90 \\
\pm 0.14\end{array}$ \\
\hline
\end{tabular}

Where

$\mathbf{C}=$ Control, $\mathbf{I M}=$ Imidacloprid, $\mathbf{H}=$ Hesperidin

Each value is the mean \pm SE, $n=6, *, a, b, c=$ Significant,

* Significant difference from Control group $(\mathrm{C}), \mathrm{P}^{*} \leq 0.05$,

a Significant difference from Imidacloprid group (IM), $\mathrm{P}^{\mathrm{a}} \leq 0.05$,

$\mathrm{b}$ Significant difference from Beta Carotene + Imidacloprid group $(\mathrm{BC}+\mathrm{IM}), \mathrm{P}^{\mathrm{b}} \leq 0.05$,

c Significant difference from Hesperidin + Imidacloprid group $(\mathrm{H}+\mathrm{IM}), \mathrm{P}^{\mathrm{c}} \leq 0.05$. 
Table (2): Protective effect of Beta Carotene and Hesperidin antioxidants against Imidacloprid insecticide induces alteration in $\alpha-F P$ and liver function tests.

\begin{tabular}{|c|c|c|c|c|c|c|c|c|c|}
\hline \multirow{3}{*}{ Group } & \multirow{3}{*}{$\mathrm{CO}$} & \multirow{2}{*}{\multicolumn{2}{|c|}{ IM }} & \multicolumn{6}{|c|}{ Antioxidants and Insecticide } \\
\hline & & & & \multicolumn{2}{|c|}{$\mathrm{BC}+\mathrm{IM}$} & \multicolumn{2}{|c|}{$\mathrm{H}+\mathrm{IM}$} & \multicolumn{2}{|c|}{$\mathrm{BC}+\mathrm{H}+\mathrm{IM}$} \\
\hline & & $1 / 10 \mathrm{LD}_{50}$ & $\begin{array}{c}1 / 5 \\
\text { LD }_{50}\end{array}$ & $1 / 10 \mathrm{LD}_{50}$ & $\begin{array}{c}1 / 5 \\
\mathrm{LD}_{50}\end{array}$ & $1 / 10 \quad \mathrm{LD}_{50}$ & $\begin{array}{c}1 / 5 \\
\text { LD }_{50}\end{array}$ & $\begin{array}{l}1 / 10 \\
\mathrm{LD}_{50}\end{array}$ & $\begin{array}{c}1 / 5 \\
\text { LD }_{50}\end{array}$ \\
\hline $\begin{array}{l}\text { AST } \\
\text { (U/L) }\end{array}$ & $\begin{array}{r}63.33 \\
\pm 2.89 \\
\end{array}$ & $\begin{array}{c}* \\
157.33 \\
\pm 5.73 \\
\end{array}$ & $\begin{array}{c}* \\
187.17 \\
\pm 10.51 \\
\end{array}$ & $\begin{array}{c}* \mathrm{a} \\
135.50 \\
\pm 6.89 \\
\end{array}$ & $\begin{array}{c}* \mathrm{a} \\
146.17 \\
\pm 5.96 \\
\end{array}$ & $\begin{array}{c}* \\
146.17 \\
\pm 6.37 \\
\end{array}$ & $\begin{array}{c}* \mathrm{a} \\
152.00 \\
\pm 5.46 \\
\end{array}$ & $\begin{array}{c}* \text { ac } \\
129.50 \\
\pm 4.87 \\
\end{array}$ & $\begin{array}{c}* \text { ac } \\
133.33 \\
\pm 5.40 \\
\end{array}$ \\
\hline $\begin{array}{l}\text { ALT } \\
\text { (U/L) }\end{array}$ & $\begin{array}{r}57.33 \\
\pm 2.78 \\
\end{array}$ & $\begin{array}{c}* * \\
121.50 \\
\pm 7.33 \\
\end{array}$ & $\begin{array}{c}* \\
135.00 \\
\pm 8.11 \\
\end{array}$ & $\begin{array}{c}*_{\mathrm{a}} \\
94.17 \\
\pm 6.41 \\
\end{array}$ & $\begin{array}{c}* a \\
110.33 \\
\pm 7.80 \\
\end{array}$ & $\begin{array}{c}*_{\mathrm{a}} \\
102.83 \\
\pm 3.42 \\
\end{array}$ & $\begin{array}{c}* a \\
114.50 \\
\pm 5.23 \\
\end{array}$ & $\begin{array}{c}* \mathrm{ac} \\
84.50 \\
\pm 2.35 \\
\end{array}$ & $\begin{array}{c}* \text { ac } \\
96.00 \\
\pm 3.71 \\
\end{array}$ \\
\hline $\begin{array}{l}\text { ALP } \\
\text { (U/L) }\end{array}$ & $\begin{array}{r}112.00 \\
\pm 5.05 \\
\end{array}$ & $\begin{array}{c}* * \\
468.00 \\
\pm 40.06 \\
\end{array}$ & $\begin{array}{c}* \\
688.00 \\
\pm 38.71 \\
\end{array}$ & $\begin{array}{c}* * \\
372.00 \\
\pm 30.78 \\
\end{array}$ & $\begin{array}{c}*{ }_{a} \\
494.00 \\
\pm 30.64 \\
\end{array}$ & $\begin{array}{c}* * \\
426.67 \\
\pm 27.41\end{array}$ & $\begin{array}{c}* \\
583.00 \\
\pm 35.50 \\
\end{array}$ & $\begin{array}{c}* a c \\
346.67 \\
\pm 19.41\end{array}$ & $\begin{array}{c}* \text { ac } \\
442.00 \\
\pm 49.26 \\
\end{array}$ \\
\hline $\begin{array}{l}\text { GGT } \\
\text { (U/L) }\end{array}$ & $\begin{array}{r}37.00 \\
\pm 2.87 \\
\end{array}$ & $\begin{array}{c}* * \\
133.00 \\
\pm 7.85 \\
\end{array}$ & $\begin{array}{c}* \\
154.00 \\
\pm 8.35 \\
\end{array}$ & $\begin{array}{c}* a \\
72.00 \\
\pm 4.19 \\
\end{array}$ & $\begin{array}{c}* \mathrm{a} \\
89.83 \\
\pm 8.78 \\
\end{array}$ & $\begin{array}{c}* a \\
105.00 \\
\pm 6.34\end{array}$ & $\begin{array}{c}* a \\
114.00 \\
\pm 9.58 \\
\end{array}$ & $\begin{array}{c}* \text { abc } \\
60.83 \\
\pm 3.07 \\
\end{array}$ & $\begin{array}{c}* a b c \\
67.00 \\
\pm 4.57 \\
\end{array}$ \\
\hline $\begin{array}{l}\text { T. Bili. } \\
\text { (mg/dl) }\end{array}$ & $\begin{array}{r}0.90 \\
\pm 0.16 \\
\end{array}$ & $\begin{array}{c}* \\
2.50 \\
\pm 0.27 \\
\end{array}$ & $\begin{array}{c}* * \\
2.60 \\
\pm 0.25 \\
\end{array}$ & $\begin{array}{c}* \mathrm{a} \\
1.50 \\
\pm 0.16 \\
\end{array}$ & $\begin{array}{c}* \mathrm{a} \\
1.80 \\
\pm 0.14 \\
\end{array}$ & $\begin{array}{c}*_{\mathrm{a}} \\
1.78 \\
\pm 0.20 \\
\end{array}$ & $\begin{array}{c}* \\
2.10 \\
\pm 0.36 \\
\end{array}$ & $\begin{array}{c}\mathrm{ac} \\
1.25 \\
\pm 0.12 \\
\end{array}$ & $\begin{array}{c}* a \\
1.60 \\
\pm 0.13 \\
\end{array}$ \\
\hline $\begin{array}{c}\alpha-\mathrm{FP} \\
(\mathrm{IU} / \mathrm{ml})\end{array}$ & $\begin{array}{c}3.60 \\
\pm 0.10 \\
\end{array}$ & $\begin{array}{c}* \\
7.70 \\
\pm 0.21 \\
\end{array}$ & $\begin{array}{c}* * \\
8.10 \\
\pm 0.36 \\
\end{array}$ & $\begin{array}{c}*{ }_{\mathrm{a}} \\
5.40 \\
\pm 0.31 \\
\end{array}$ & $\begin{array}{c}* \mathrm{a} \\
6.25 \\
\pm 0.41 \\
\end{array}$ & $\begin{array}{c}* a \\
6.10 \\
\pm 0.35 \\
\end{array}$ & $\begin{array}{c}* a \\
6.60 \\
\pm 0 . .22 \\
\end{array}$ & $\begin{array}{c}\text { *abc } \\
4.98 \\
\pm 0.25 \\
\end{array}$ & $\begin{array}{c}\text { *abc } \\
5.70 \\
\pm 0.29 \\
\end{array}$ \\
\hline
\end{tabular}

Where

$\mathrm{C}=$ Control, $\mathrm{IM}=$ Imidacloprid, $\mathrm{H}=$ Hesperidin

Each value is the mean $\pm \mathrm{SE}, \mathrm{n}=6,{ }^{*}, \mathrm{a}, \mathrm{b}, \mathrm{c}=$ Significant,

* Significant difference from Control group $(\mathrm{C}), \mathrm{P}^{*} \leq 0.05$,

a Significant difference from Imidacloprid group (IM), $\mathrm{P}^{\mathrm{a}} \leq 0.05$,

b Significant difference from Beta Carotene + Imidacloprid group $(\mathrm{BC}+\mathrm{IM}), \mathrm{P}^{\mathrm{b}} \leq 0.05$,

c Significant difference from Hesperidin + Imidacloprid group $(\mathrm{H}+\mathrm{IM}), \mathrm{P}^{\mathrm{c}} \leq 0.05$.

Table (3): Protective effect of Beta Carotene and Hesperidin antioxidants against Imidacloprid insecticide induces alteration in T.P., Albumin, T. Chol. and T.G. concentrations.

\begin{tabular}{|c|c|c|c|c|c|c|c|c|c|}
\hline \multirow{3}{*}{ Group } & \multirow{3}{*}{$\mathrm{CO}$} & \multirow{2}{*}{\multicolumn{2}{|c|}{ IM }} & \multicolumn{6}{|c|}{ Antioxidants and Insecticide } \\
\hline & & & & \multicolumn{2}{|c|}{$\mathrm{BC}+\mathrm{IM}$} & \multicolumn{2}{|c|}{ H+IM } & \multicolumn{2}{|c|}{$\mathrm{BC}+\mathrm{H}+\mathrm{IM}$} \\
\hline & & $\begin{array}{l}1 / 10 \\
\text { LD } 50\end{array}$ & $\begin{array}{c}1 / 5 \\
\text { LD }_{50}\end{array}$ & $1 / 10 \mathrm{LD}_{50}$ & $\begin{array}{l}1 / 5 \\
\text { LD }_{50}\end{array}$ & $1 / 10 \quad$ LD $_{50}$ & $\begin{array}{l}1 / 5 \\
\text { LD } 50\end{array}$ & 1/10 LD50 & $\begin{array}{c}1 / 5 \\
\text { LD }_{50}\end{array}$ \\
\hline $\begin{array}{c}\text { TP } \\
\text { (g/dl) }\end{array}$ & $\begin{array}{c}7.20 \\
\pm 0.24 \\
\end{array}$ & $\begin{array}{c}* \\
5.30 \\
\pm 0.27 \\
\end{array}$ & $\begin{array}{c}* \\
4.37 \\
\pm 0.15 \\
\end{array}$ & $\begin{array}{c}6.20 \\
\pm 0.44 \\
\end{array}$ & $\begin{array}{c}* \\
5.10 \\
\pm 0.36 \\
\end{array}$ & $\begin{array}{c}* \\
5.88 \\
\pm 0.36 \\
\end{array}$ & $\begin{array}{c}* \\
4.80 \\
\pm 0.31 \\
\end{array}$ & $\begin{array}{c}\mathrm{a} \\
6.78 \\
\pm 0.29 \\
\end{array}$ & $\begin{array}{c}* \mathrm{ac} \\
6.00 \\
\pm 0.43 \\
\end{array}$ \\
\hline $\begin{array}{l}\text { Alb. } \\
\text { (g/dl) }\end{array}$ & $\begin{array}{c}4.50 \\
\pm 0.34 \\
\end{array}$ & $\begin{array}{c}* \\
3.30 \\
\pm 0.31 \\
\end{array}$ & $\begin{array}{c}* \\
3.00 \\
\pm 0.90 \\
\end{array}$ & $\begin{array}{r}3.90 \\
\pm 0.29 \\
\end{array}$ & $\begin{array}{c}\mathrm{a} \\
3.70 \\
\pm 0.27 \\
\end{array}$ & $\begin{array}{r}3.78 \\
\pm 0.16 \\
\end{array}$ & $\begin{array}{c}* \\
3.40 \\
\pm 0.20 \\
\end{array}$ & $\begin{array}{c}\mathrm{a} \\
4.27 \\
\pm 0.22 \\
\end{array}$ & $\begin{array}{c}\mathrm{a} \\
3.93 \\
\pm 0.24 \\
\end{array}$ \\
\hline $\begin{array}{l}\text { T. Chol } \\
(\mathrm{mg} / \mathrm{dl})\end{array}$ & $\begin{array}{l}91.17 \\
\pm 4.19 \\
\end{array}$ & $\begin{array}{c}* \\
110.00 \\
\pm 5.76 \\
\end{array}$ & $\begin{array}{c}* * \\
131.18 \\
\pm 2.30 \\
\end{array}$ & $\begin{array}{r}98.00 \\
\pm 3.06 \\
\end{array}$ & $\begin{array}{c}102.50 \\
\pm 5.56 \\
\end{array}$ & $\begin{array}{c}105.00 \\
\pm 4.24 \\
\end{array}$ & $\begin{array}{c}115.00 \\
\pm 6.04 \\
\end{array}$ & $\begin{array}{c}\mathrm{a} \\
92.50 \\
\pm 2.68 \\
\end{array}$ & $\begin{array}{c}\mathrm{a} \\
98.00 \\
\pm 4.56 \\
\end{array}$ \\
\hline $\begin{array}{c}\mathrm{TG} \\
(\mathrm{mg} / \mathrm{dl})\end{array}$ & $\begin{array}{l}54.00 \\
\pm 2.49\end{array}$ & $\begin{array}{c}* * \\
98.17 \\
\pm 2.44\end{array}$ & $\begin{array}{c}* \\
112.08 \\
\pm 4.50\end{array}$ & $\begin{array}{c}* * \\
83.00 \\
\pm 2.56\end{array}$ & $\begin{array}{c}* \\
95.00 \\
\pm 2.60\end{array}$ & $\begin{array}{c}* * \\
91.83 \\
\pm 2.53\end{array}$ & $\begin{array}{c}* \\
100.45 \\
\pm 4.27\end{array}$ & $\begin{array}{c}* \text { abc } \\
67.40 \\
\pm 2.18\end{array}$ & $\begin{array}{c}* \\
89.33 \\
\pm 3.80\end{array}$ \\
\hline
\end{tabular}

Where

$\mathrm{C}=\mathrm{Control}, \mathrm{IM}=$ Imidacloprid, $\mathrm{H}=$ Hesperidin

Each value is the mean \pm SE, $n=6, *, a, b, c=$ Significant,

* Significant difference from Control group $(\mathrm{C}), \mathrm{P} * \leq 0.05$,

a Significant difference from Imidacloprid group (IM), $\mathrm{P}^{\mathrm{a}} \leq 0.05$,

$\mathrm{b}$ Significant difference from Beta Carotene + Imidacloprid group $(\mathrm{BC}+\mathrm{IM}), \mathrm{P}^{\mathrm{b}} \leq 0.05$,

c Significant difference from Hesperidin + Imidacloprid group $(\mathrm{H}+\mathrm{IM}), \mathrm{P}^{\mathrm{c}} \leq 0.05$. 


\section{Fig. 1}

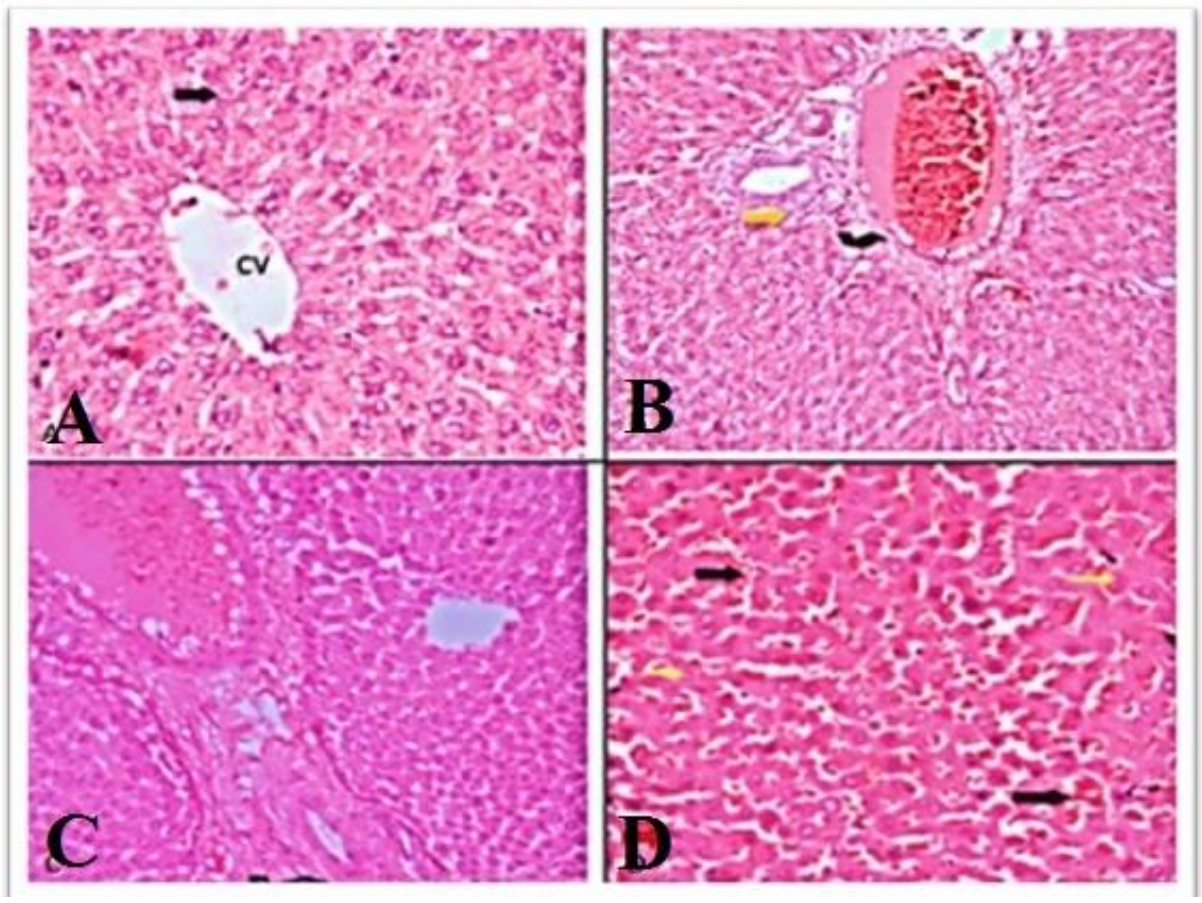

Fig.1: Sections of the liver of rats (A): control showing normal histological structure of hepatic lobules and central vein(cv) $(\mathrm{Hx} \& \mathrm{E} \times 400)(\mathbf{B})$ : treated with 1/5 LD50 of imidacloprid insecticide showing dilated, congested, edematous, vacuolated portal vein(black curved arrow) with infiltration of leukocytes and a fibrosis around the bile duct (yellow arrow). (Hx\&E x100) (C): High power of the previous section showing fibrosis around bile duct (Hx\&E x400) (D): Another field of the liver of rat of the same previous group showing Dilated and congested blood sinusoid (black arrow), sings of degeneration in the form of karyolysis (yellow arrow), karyorhexis (red arrow) and foci of necrosis were seen.(HX\&E x400).

\section{Fig. 2}

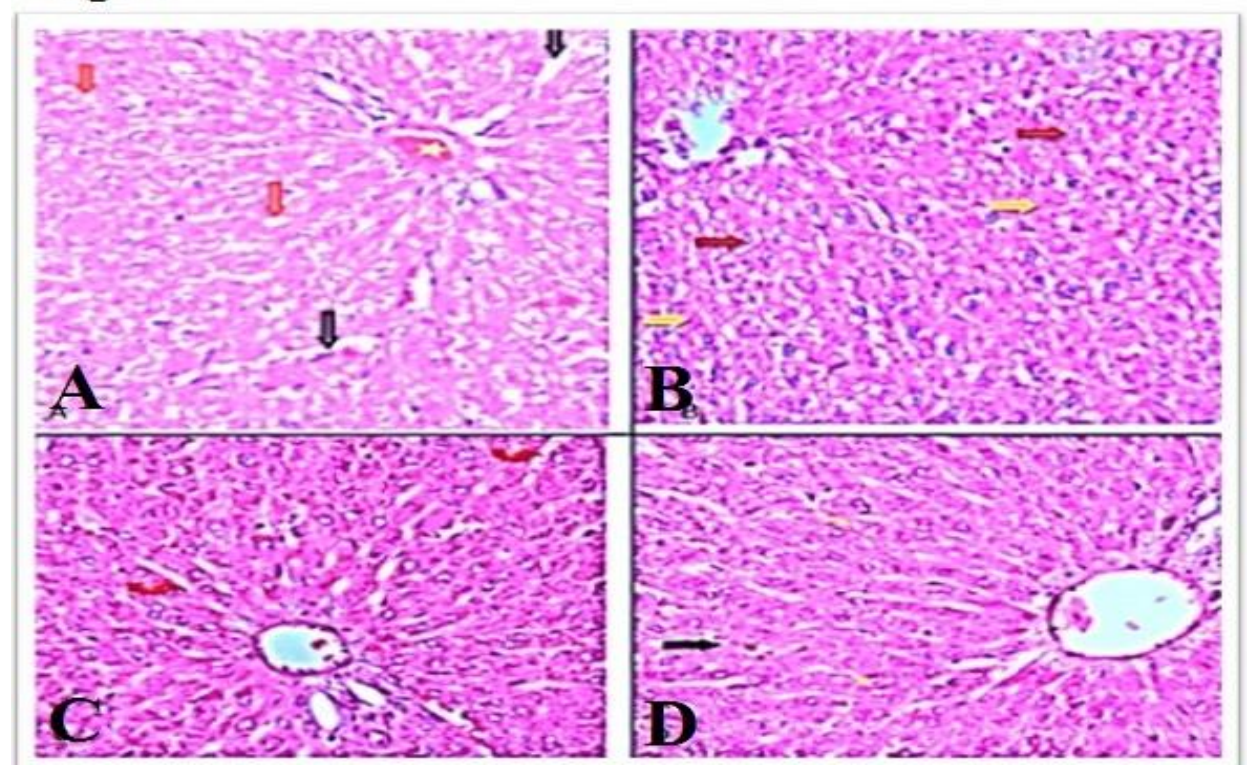

Fig1: Sections of the liver of rats (A): treated with $1 / 10 L D_{50}$ of imidacloprid insecticide showing congestion of portal vein and thickening of the wall (star), dilated blood sinusoid (black arrow) and vacuolar degeneration (red arrow). (HX\&E X400) (B): Another field of the liver of rat of the same previous group showing foci of necrosis (yellow arrow) and karyolysis ( $H \times \& E \times 400)(C)$ : treated with $1 / 5 L_{50}$ of imidacloprid insecticide along with beta carotene showing normal histological structure of hepatic lobules but mild dilated of blood sinusouid (red arrow) vacuolar degeneration (black arrow) (Hx\&E x400) (D): rat treated with $1 / 10 L_{50}$ of imidacloprid insecticide along with beta carotene showing normal histological structure of hepatic lobules but mild dilated and congestion of blood sinusouid (black arrow)and minute vacuolar degeneration (yellow arrow) .(Hx\&E x400). 


\section{Fig. 3}

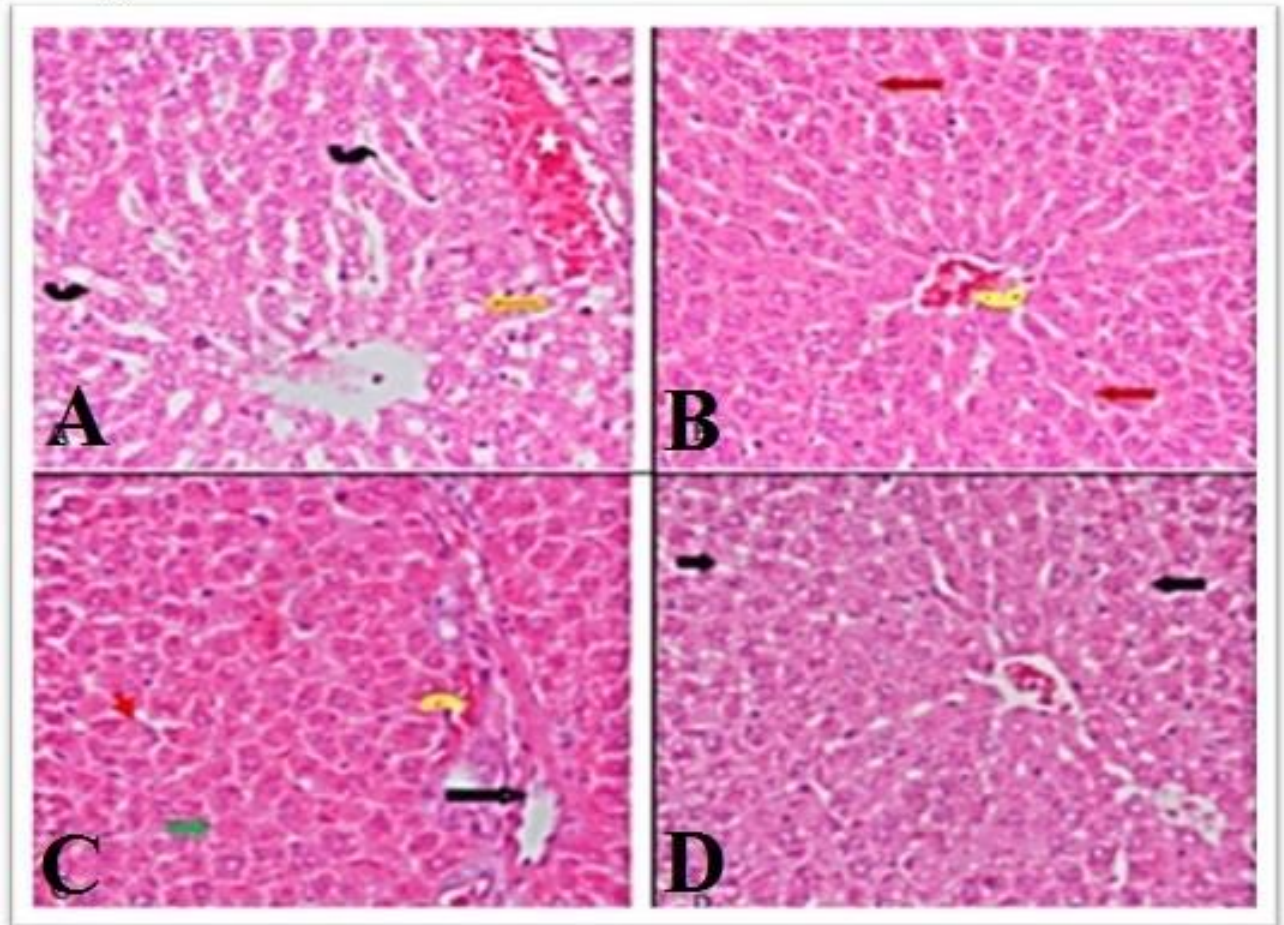

Fig.3: Sections of the liver of rats (A): treated with $1 / 5 \mathrm{LD}_{50}$ of imidacloprid insecticide along with hesperidin showing vacuolar degeneration (yellow arrow), dilated blood sinusoid (black arrow) and dilated and congested blood vessel (star). (Hx\&E x400) (B): treated with 1/10 $\mathrm{LD}_{50}$ of imidacloprid insecticide along with hesperidin showing most of hepatocyte appeared normal few appeared necrotic (red arrow) and congested blood vessel (yellow arrow). (Hx\&E x200) (C): treated with 1/5 LD 50 of imidacloprid insecticide along with beta carotene and hesperidin showing most of hepatocyte appeared normal but necrosis of some hepatocyte (green arrow) thickening in the wall of portal vessel (black arrow), hypertrophied of kupffer cells(red arrow). (Hx\&E x400) (D): treated with $1 / 10 \mathrm{LD}_{50}$ of imidacloprid insecticide along with beta carotene and hesperidin showing most of hepatocyte appeared normal but minute vacuolar degeneration still present (black arrow). (Hx\&E x200).

\section{DISCUSSION}

Exposure to imidacloprid has been associated with hepatotoxicity and tissue damage (Arfat et al., 2014) through several oxidative mechanisms and lipid peroxidation that modulate antioxidants or free radical scavenging enzyme systems (El-Gendy et al., 2010). Oxidative damage may result from decreased clearance of ROS by scavenging mechanisms. The cells are provided with a number of mechanisms to protect themselves from the toxic effects of oxidative stress. GSH is highly abundant in all cell compartments and is the major soluble antioxidant. GSH shows its antioxidant effects in several ways. It detoxifies hydrogen peroxide and lipid peroxides. GSH donates its electron to $\mathrm{H} 2 \mathrm{O} 2$ to reduce it into
$\mathrm{H} 2 \mathrm{O}$ and $\mathrm{O} 2$. GSSG is again reduced into GSH by GSH reductase that uses $\mathrm{NAD}(\mathrm{P}) \mathrm{H}$ as the electron donor (Masella et al., 2005). The SOD eliminates superoxide by converting it into $\mathrm{H}_{2} \mathrm{O}_{2}$ which is rapidly transformed to water by CAT (Mahdi, 2002). Our results showed that imidacloprid motivates oxidative stress as evidenced by an increase of hepatic MDA, marker of lipid peroxidation and a decrease of GSH, CAT and SOD levels. The decreased activity of CAT, SOD (antioxidant enzymes) and GSH along with an increase of lipid peroxide levels could likely be associated with oxidative stress and decreased antioxidant defense potential. The decreased rate of glutathione synthesis has been found in many diseases such as fibrosis and a variety of hepatic disorders. Decreased GSH synthesis 
occurs as a result of decreased expression of the synthetic enzymes (Lu, 2013). Since GSH is involved in the removal of reactive oxygen species (ROS) from the cells, a decreased production of GSH will lead to an accumulation of ROS in the cell that cause DNA, protein and membrane damage (Anderson, 1998). The hepatic N.O elevated significantly in the present investigation due to IM treatment. Despite nitric oxide has important physiological functions, N.O is also a well-known toxic agent (Grisham et al., 1999). Overproduction of N.O in the liver has been involved as an important incident in hepatic inflammation and injury (Gardner et al., 1998).

Treatment with $\beta$-carotene or hesperidin to IM given rats lowered hepatic MDA and increased hepatic GSH, CAT, and SOD levels as compared to IM group. This may be referred to antioxidant property of $\beta$-carotene or hesperidin, which scavenges the free radical and thereby, maintain the levels of antioxidants (GSH, CAT, SOD).The antioxidant mechanism of $\beta$-carotene and hesperidin has been suggested to occur via quenching of singlet oxygen, free radical scavenging and chain breaking during lipid peroxidation (Galano et al., 2010 and Balakrishnan \& Menon, 2007). $\beta$-carotene Protects of cellular membranes and lipoproteins against oxidative damage (Ciccone et al., 2013). Carotenoids quench singlet oxygen primarily by a physical mechanism, in which excess energy of single oxygen is transferred to carotenoids and then they return into the ground state, as a result carotenoids offer to protect against further oxygen radical and lipid peroxidation (Mc Nulty et al., 2008). Both $\beta$-carotene and hesperidin can combat oxidative stress. $\beta$-carotene reduced oxidative stress evoked by acetaminophen and restored the activity of hepatic antioxidant defense system (Morakinyo et al., 2012). Hesperidin attenuates cyclophosphamide induced depletion of cardiac antioxidant enzymes and elevation of MDA level (Kumar et al., 2011).
The present results demonstrated a significant increase in serum liver enzymes, bilirubin and alpha-fetoprotein. Some studies showed that AST elevation was significantly associated with the rise of AFP level (Chen et al., 2008 and $\mathrm{Hu}$ et al., 2004). The liver damage and change of membrane characteristics led to the leakage of hepatic enzymes and therefore elevation of its level in the plasma. The increase in liver enzymes may indicate hepatocytes necrosis (Gressner et al., 2007). The histopathological changes in the liver induced by IM affirm the previous results as manifested by fibrosis around the bile duct, vacuolar degeneration, dilated congested blood sinusoid, and necrosis of some hepatocytes. The results of imidacloprid group are dose dependent. The histopathological changes can be attributed to increasing of hepatic MDA level, a marker of lipid peroxidation or TNF- $\alpha$. Lipid peroxidation is a process under which oxidants such as free radicals attack lipids, particularly polyunsaturated fatty acids that involve hydrogen abstraction from a carbon, with oxygen insertion resulting in lipid peroxyl radicals and hydroperoxides as described previously (Szabó et al., 2007). The lipid free radical interactions produce peroxides, which are themselves unstable and reactive and an autocatalytic chain reaction called propagation which can result in an extensive membrane, organellar, and cellular damage (Kumar et al., 2009). It was reported that the treatment of rats with imidacloprid led to a rise in liver enzymes, oxidative stress and histopathological changes in liver (Soujanya et al., 2013 and Mohany et al., 2012). It was suggested that the oxidative stress and elevated level of TNF- $\alpha$ play an essential role in hepatic cell injury (Hazzaa et al., 2013).

Concerning the essential role of oxidative stress in liver diseases, some antioxidants are suggested to prevent and treat liver diseases ( $\mathbf{L i}$ et al., 2014 and Medina \& Moreno-Otero, 2005). The effectiveness of some antioxidants in the treatment of patients with various liver diseases, such as chronic hepatitis has been studied. The clinical effects of antioxidants on 
chronic hepatitis $\mathrm{C}$ patients have shown a clear benefit of antioxidants as interferon based therapy of hepatitis (Esrefoglu et al., 2012). In the present study, the treatment with betacarotene or hesperidin significantly reduced the hepatotoxic effect of imidacloprid, this was shown by the significant decrease in serum liver enzymes and bilirubin.Moreover, they minimized the histopathological changes induced by IM. The hepatoprotective effects of beta-carotene or hesperidin can be attributed to their ability to lower lipid peroxidation and enhancement of antioxidant defense system. Many reports indicated a hepatoprotective effect of $\beta$-carotene and hesperidin via reducing oxidative stress. $\beta$-carotene reduced acetaminophen and cadmium induced liver damage (Morakinyo et al., 2012 and Bashandy \& Alhazza, 2008). Hesperidin may be promising protective agent against liver fibrosis through improvement of liver function, modulation of the fibrous scar formation, antiinflammatory and antioxidant potentials (Abdel-Sttar et al., 2017).

$\beta$-carotene, and hesperidin lowered the increase of serum AFP and GGT evoked by IM. AFP and GGT are markers of hepatocellular carcinoma (Zhao et al., 2013). Hesperidin inhibits the growth of human hepatocarcinoma HepG2 cell line through suppressing the activity of NF-kB gene and modulates the biochemical marker AFP (Fathy et al., 2014). The characteristic of $\beta$-carotene (BC) as an efficient antioxidant plays an important role in decreasing the incidence of cancer (Riboli and Norat, 2003).

There was an association between alphafetoprotein levels and increased risk for metabolic syndrome including a rise of cholesterol and triglycerides levels (Chen et al., 2016). The elevation of serum triglycerides can be referred to a suppression of the lipase enzyme activity (Goldberg et al., 1982) or to lipolysis stimulation by TNF- $\alpha$ (Sethi et al., 2000). It was found that TNF- $\alpha$ increases plasma TG by increasing the concentration of free fatty acids (Feingold et al., 1994), the substrate for TG synthesis. Both beta-carotene and hesperidin inhibit the elevation of cholesterol and triglycerides induced by IM in rats. It was found that Dietary supplementation with beta-carotene decreased serum total cholesterol and hepatic total lipid and cholesterol contents .These changes were accompanied by an increase in the total lipid and cholesterol contents excreted in the feces (Silva et al., 2013). The previous author suggested that beta-carotene administration may decrease cholesterol absorption in the intestine and increase cholesterol excretion into the feces without a direct effect on the expression of cholesterol metabolism genes. Bok et al. (1999) concluded that hesperidin significantly lowered the plasma and hepatic levels of cholesterol and TG by inhibiting HMG-CoA reductase and acyl CoA: cholesterol acyltransferase in rats.

A synergistic effect of a combination of hesperidin, a flavonoid, and Lycopene, a carotenoid has been reported due to their antioxidant activities (Jain and Katti, 2015). In the present study, the synergetic potential of the combination of $\beta$-carotene and hesperidin appears beneficial in the mitigation of hepatotoxicity induced by IM.

In conclusion, chronic imidacloprid treatment gives rise to oxidative stress and inflammation by modifying antioxidant systems and motivating pro-inflammatory cytokine production in the hepatic cells. Both $\beta$-carotene, and hesperidin reduced the deleterious effects of imidacloprid on liver via allevation of oxidative stress, reduction of inflammatory cytokine level and maintaining antioxidant enzymes. The present study proposed a synergistic effect of a combination of $\beta$ carotene and hesperidin.

\section{Conflict of interest:}

The authors have declared that no competing interests exist.

\section{REFERENCES}


Abdel-Sttar, A.R.; Khalaf, M.M.; Abo-youssef, A.M. and Abo-saif, A. (2017): Ameliorative effect of hesperidin on carbon tetrachloride induced liver fibrosis in rats. Int. J. Pharm. Pharm. Sci., 9(7): 45-51.

Agarwal, R. and Srinivas, R. (2007): Severe neuropsychiatric manifestations and rhabdomyolysis in a patient with imidacloprid poisoning. Am. J. Emerg. Med., 25: 844-845. Researchgate.net/5971233.

Ahmed, M.M. and Nasr, S.A. (2015): Protective effect of broccoli and ferulic acid on imidacloprid-induced neurotoxicity in rats. J. Biomed. Pharm. Res., 4(4): 82-89.

Ahmed, R.R.; Mahmoud, A.M.; Ashour, M.B. and Kamel, A.M. (2015): Hesperidin protects against diethylnitrosamine-induced nephrotoxicity through modulation of oxidative stress and inflammation. Natl. J. Physiol. Pharm. Pharmacol., 5(5):391-397.

Akiyama, S.; Katsumata, S.; Suzuki, K.; Nakaya, Y.; Ishimi, Y. and Uehara, M. (2009): Hypoglycemic and Hypolipidemic Effects of Hesperidin and Cyclodextrin-Clathrated Hesperetin in Goto-Kakizaki Rats with Type 2 Diabetes. Biosci. Biotechnol. Biochem., 73(12): 2779-2782. PMID: 19966469.

Anderson, M.E. (1998): Glutathione: An overview of biosynthesis and modulation. Chem. Biol. Interact., 111-112: 1-14. PMID: 9679538.

Ansari, M.A.; Keller, J.N. and Scheff, S.W. (2008): Protective effect of Pycnogenol in human neuroblastoma SH-SY5Y cells following acrolein-induced cytotoxicity. Free Radic. Biol. Med., 45: 1510-1519. PMID: 18822368 .

Arfat, Y.; Mahmood, N.; Tahir, M.U.; Rashid, M.; Anjum, S. and Zhao, F. (2014): Effect of imidacloprid on hepatotoxicity and nephrotoxicity in male albino mice. Toxicology Reports, 1: 554-561. science/article/pii/S2214750014000675.

Balakrishnan, A. and Menon, V.P. (2007): Antioxidant properties of hesperidin in nicotine-induced lung toxicity. Fundam. Clin. Pharmacol., 21: 535-546. PMID: 17868207.

Bashandy, S.A. and Alhazza, I.M. (2008): The hepatoprotective of $\beta$-carotene against cadmium toxicity in rats. J. Pharm. Toxicol., 3(6): 457-463. scialert.net/doi.2008.457.463

Bok, S.H.; Lee, S.H.; Park, Y.B.; Bae, K.H.; Son, K.H.; Jeong, T.S. and Choi, M.S. (1999): Plasma and hepatic cholesterol and hepatic activities of 3-hydroxy-3-methyl-glutaryl-CoA reductase and acyl CoA: cholesterol transferase are lower in rats fed citrus peel extract or a mixture of citrus bioflavonoids. J. Nutr., 129: 1182-1185. PMID: 10356084.

Chen, C.H.; Lin, S.T.; Kuo, C.L. and Nien, C.K. (2008): Clinical significance of elevated alphafetoprotein (AFP) in chronic hepatitis $\mathrm{C}$ without hepatocellular carcinoma. Hepatogastroenterology, 55: 1423-1427. PMID: 18795704

Chen, Y.; Zhao, Y.; Feng, L.; Zhang, J.; Zhang, J. and Feng, G. (2016): Association between alpha-fetoprotein and metabolic syndrome in a Chinese asymptomatic population: a crosssectional study. Lipids in Health and Disease, 15(85): 2-9. Lipid world.biomed central/12944016-0256.

Ciccone, M.M.; Cortese, F.; Gesualdo, M.; Carbonara, S.; Zito, A. and Ricci, G. (2013): Dietary Intake of Carotenoids and Their Antioxidant and Anti-Inflammatory Effects in Cardiovascular Care. Mediators of Inflammation, 2013: 1-11. PMID: 24489447.

Cui, B.; Liu, S.; Wang, Q. and Lin, X. (2012): Effect of $\beta$-Carotene on immunity function and tumour growth in hepatocellular carcinoma rats. PLoS One, 17: 8595-8603. PMID: 22810193.

Devi, K.P.; Rajavel, T.; Nabavi, S.F.; Setzer, W.N.; Ahmadi, A.; Mansouri, K. and Nabavi, S.M. (2015): Hesperidin: A promising anticancer agent from nature. Industrial crops and products, 76: 582-589. Researchgate.net/280599310.

El-Gendy, K.S.; Aly, N.M.; Mahmoud, F.H.; Kenawy, A. and El-Sebae, A.K. (2010): The 
role of vitamin $\mathrm{C}$ as antioxidant in protection of oxidative stress induced by imidacloprid. Food chem. Toxicol., 48: 215-221. PMID: 19833166.

Esrefoglu, M. (2012): Oxidative stress and benefits of antioxidant agents in acute and chronic hepatitis. Hepat. Mon., 12: 160-167. PMID: 3339415

Fathy, S.A.; Abdel-Hamid, F.F.; Agwa, S.A. and El-Diasty, S.M. (2014): The Anti-Proliferative Effect of Hesperidin on Hepatocarcinoma Cells HepG2. Egypt. Acad. J. Biolog. Sci., 6(2): 75 83.

Feingold, K. R.; Marshall, M.; Gulli, R.; Moser, A. H. and Grunfeld, C. (1994): Effect of endotoxin and cytokines on lipoprotein lipase activity in mice. Arterioscler. Thromb., 14: 1866-1872. PMID: 7947614.

Felsot, A. (2001): Admiring risk reduction. Agrichemical Environmental News, p. 186.

Galano, A.; Vargas, R. and Martinez, A. (2010): Carotenoids can act as antioxidants by oxidizing the superoxide radical anion. Phys. Chem. Chem. Phys., 12: 193-200. PMID: 20024459.

Gardner, C.R.; Heck, D.E.; Yang, C.S.; Thomas, P.E.; Zhang, X.J. and De-George, G.L. (1998): Role of nitric oxide in acetaminopheninduced hepatotoxicity in the rat. Hepatology, 27:748-754. PMID: 9500703.

Goldberg, I.J.; Le, N.A.; Paterniti, J.R.; Ginsberg, H.N.; Lindgren, F.T. and Brown, W.V. (1982): Lipoprotein metabolism during acute inhibition of hepatic triglyceride lipase in the Cynomolgus monkey. J. Clin. Invest., 70(6): 1184-1192. PMID: 7174789.

Gressner, O.A.; Weiskirchen, R. and Gressner, A.M. (2007): Biomarkers of liver fibrosis: clinical translation of molecular pathogenesis or based on liver-dependent malfunction tests. Clin. Chim. Acta., 381(2): 107-113. PMID: 17399697.

Grisham, M.B.; Jourd-Heuil, D. and Wink, D.A. (1999): Nitric Oxide I. Physiological chemistry of nitric oxide and its metabolites: implications in inflammation. Am. J. Physiol. Gastrointest. Liver Physiol., 276(2): G315-G321. PMID: 9950804.

Hazzaa, S.; Badr, E. and Abdou, A. (2013): The link between oxidative stress response and tumor necrosis factor-alpha (TNF-alpha) in hepatic tissue of rats with induced thyroid dysfunction. J. Afr. Ass. Physiol. Sci., 1 (1): 47 - 54.jaaps/article/ 132644.

Hozayen, W.G.; Abou-Seif, H.S. and Amin, S. (2014): Protective Effects of Ruitn and / or Hesperidin Against Doxorubicin-Induced Hepatotoxicity. Inter. J. Clin. Nutr., 2(1): 11-17. Researchgate.net/268347236.

Hu, K.Q.; Kyulo, N.L.; Lim, N.; Elhazin, B.; Hillebrand, D.J. and Bock, T., (2004): Clinical significance of elevated alphafetoprotein (AFP) in patients with chronic hepatitis $\mathrm{C}$, but not hepatocellular carcinoma. Am. J. Gastroenterol., 99: 860-865. PMID: 18233991.

Hutchinson, M.J.; Jacobs, D.E. and Mencke, N. (2001): Establishment of the cat flea (Ctenocephalides felis felis) on the ferret (Mustela putorius furo) and its control with imidacloprid. Med. Veterinary Entomology, 15 (2): 212-214. PMID: 11434557.

Li, A.N.; Li, S.; Zhang, Y.J.; Xu, X.R.; Chen, Y.M. and Li, H.B. (2014): Resources and biological activities of natural polyphenols. Nutrients, 6: 6020-6047. PMID: 25533011.

Lorke, D. (1983): A new approach to acute toxicity testing. Arch. Toxicol., 54(4): 275-287. PMID: 6667118.

Jain, D. and Katti, N. (2015): Combination treatment of lycopene and hesperidin protect experimentally induced ulcer in laboratory rats. J. Intercult. Ethnopharmacol., 4(2): 143146.PMID: 26401402.

Kensler, T.W.; Wakabayashi, N.B. and Iswal, S. (2007): Cell survival responses to environmental stresses via the Keap1-Nrf2- 
ARE pathway. Annu. Rev. Pharmacol. Toxicol., 47: 89-116. PMID: 16968214.

Kheir-Eldin, A.A.; Motawi-Nermin, T.M.K. and Sadik. A.H. (2008): Effect of some natural antioxidants on aflatoxin B1-induced hepatic toxicity. EXCLI Journal, 7: 119-131. Researchgate.net/28357972.

Kumar, V.; Abbas, A.K. and Fausto, N. (2009): Cellular Adaptations, cell injury and cell death. In: Pathologic basis of disease. Robbins and Cotran, editors. 8 th ed. Philadelphia: Saunders, p. 15-18.

Kumar, S.; Dhankhar N.; Kar,V.; Shrivastava, M. and Shrivastava, S. (2011): Myocardial Injury Provoked by Cyclophosphamide, Protective Aspect of Hesperidin in Rats. International Journal of Research in Pharmaceutical and Biomedical. Sciences, 2(3): 1288-1296. Researchgate.net/268285994.

Kumar, B.H.; Kumar, B.D. and Diwan, P.V. (2017): Hesperidin, a citrus flavonoid, protects against L-methionine-induced hyperhomocysteinemia by abrogation of oxidative stress, endothelial dysfunction and neurotoxicity in Wistar rats. Pharmaceutical Biology, 55(1): 146-155. Researchgate.net/308762664.

Levaj, B.; Dragovic-Uzelac, V.; Kovacvic, D.B. and Krasnici, N. (2009): Determination of Flavonoids in Pulp and Peel of Mandarin Fruits. Agric. conspec. sci., 74(3): 221-225.

Lu, S.C. (2013): Glutathione Synthesis. Biochim. Biophys. Acta., 1830(5): 3143-3153. PMID: 3549305.

Mahdi, A.A. (2002): Free radicals and other antioxidants. In: A Text Book of Biochemistry. 3rd ed. Edited by Singh SP, New Dehli: CBS Publishersand Distributors, pp. 545-555.

Mahmoud, A. M. (2014): Hesperidin protects against cyclophosphamide-induced hepatotoxicity by upregulation of PPAR $\gamma$ and abrogation of oxidative stress and inflammation. Can. J. Physiol. Pharmaco., 92(9): 717-724.Researchgate.net/263083202.
Masella, R.; Di-Benedetto, R.; Vari, R.; Filesi, C. and Giovannini, C. (2005): Novel mechanisms of natural antioxidant compounds in biological systems: involvement of glutathione and glutathione-related enzymes. J. Nutr. Biochem., 16: 577-586. PMID: 16111877.

Mc-Nulty, H.; Jacob, R.F. and Mason, R.P. (2008): Biological activity of carotenoids related to distinct membrane physiochemical interactions. Am. J. Cardiol., 101: 20D-29D. PMID: 18474269.

Medina, J. and Moreno-Otero, R. (2005): Pathophysiological basis for antioxidant therapy in chronic liver disease. Drugs, 65(17): 24452461. PMID: 16296871

Mohany, M.; Badr, G.; Refaat, I.; Garraud, O. and EI-feki, M. (2012): Thymoquinone ameliorates immunological and histological changes induced by exposure to imidacloprid insecticide. Toxicology Science, 37(1): 1-11. PMID: 22293407.

Morakinyo, A.O.; Iranloye, B.O.; Oyelowo, O.T. and Nnaji, J. (2012): Anti-oxidative and hepatoprotective effect of beta-carotene on acetaminophen-induced liver damage in rats. Biology and Medicine, 4(3): 134-140.

Novo, R.; Azevedo, P.S.; Minicucci, M.F.; Zornoff, L.A.M. and Paiva, S.A.R. (2013): Effect of Beta-Carotene on Oxidative Stress and Expression of Cardiac Connexin 43. Arq. Bras. Cardiol., 101(3): 233-239. PMID: 23917457.

Orhan, I.; Ozcelik, B. and Şener, B. (2011): Evaluation of antibacterial, antifungal, antiviral, and antioxidant potentials of some edible oils and their fatty acid profiles. Turk. J. Biol. 35: 251-258. Researchgate.net/228504836.

Proenca, P.; Teixeira, H.; Castanheira, F.; Pinheiro, J.; Monsanto, P.V.; Marques, E.P. and Vieira, D.N. (2005): Two fatal intoxication cases with imidacloprid: LC/MS analysis. Forensic Sci. Int., 153: 75-80. PMID: 15922528.

Riboli, E. and Norat, T. (2003): Epidemiologic evidence of the protective effect of fruit and 
vegetables on cancer risk. Am. J. Clin. Nutr., 78: 559S-569S. PMID: 12936950.

Sethi, J.K.; Xu, H.; Uysal, K.T.; Wiesbrock, S.M.; Scheja, L. and Hotamisligil, G.S. (2000): Characterisation of receptor-specific TNF-alpha functions in adipocyte cell lines lacking type 1 and 2 TNF receptors. FEBS Lett., 469: 77-82. PMID: 10708760.

Shi, J.; Kakuda, Y. and Yeung, D. (2004): Antioxidative properties of lycopene and other carotenoids from tomatoes: synergistic effects. Biofactors, 21(1-4): 203-210. PMID: 15630198.

Silva, L.S.; de-Miranda, A.M.; de BritoMagalhães, C.L.; dos-Santos, R.C.; Pedrosa, M.L. and Silva, M.E. (2013): Diet supplementation with beta-carotene improves the serum lipid profile in rats fed a cholesterolenriched diet. J. Physiol. Biochem., 69: 811820. PMID: 23645541.

Soujanya, S.; Lakshman, M.; Anand-Kumar, A. and Gopala-Reddy, A. (2013): Evaluation of the protective role of vitamin $\mathrm{C}$ in imidacloprid induced hepatotoxicity in male albino rats. J. Nat. Sci. Biol. Med., 4(1): 63-67. PMID: 23633837.

Stahl, W. and Sies, H. (2003): Antioxidant activity of carotenoids. Mol. Aspects Med., 24(6): 345351. PMID: 14585305.

Stephan, B.; Kyle, L. and Yong, X. (1997): Role of oxidative stress in the mechanism of dieldrin's hepatotoxicity. Ann. Clin. Lab. Sci., 27: 196-208. PMID: 9142372.

Szabo, C.; Ischiropoulos, H. and Radi, R. (2007): Peroxynitrite: biochemistry, pathophysiology and development of therapeutics. Nat. Rev. Drug. Discov., 6(8): 662-680. PMID: 17667957. Biomedica., 25: 171-174.

Vabeiryureilai, M.; Lalrinzuali, K. and Jagetia, G.C. (2015): Determination of antiinflammatory and analgesic activities of a citrus bioflavonoid, hesperidin in Mice. Immunochem. Immunopathol., 1(2): 17.Researchgate.net/15177045.
Whitehorn, P.R.; Connor, S.; Wackers, F.L. and Goulson, D. (2012): Neonicotinoid pesticide reduces bumble bee colony growth and queen production. Science, 336: 351352.Researchgate.net/267525648.

Wilmsen, P.K.; Spada, D.S. and Salvador, M. (2005): Antioxidant activity of the flavonoid hesperidin in chemical and biological systems. J. Agric. Food Chem., 15: 4757-4761._PMID: 15941311.

Zhao, Y-J.; Ju, Q. and Li, G-C. (2013): Tumor markers for hepatocellular carcinoma (Review). Mol. Clin. Oncol., 1: 593-598. PMID: 3915636. 